\title{
Risque viral lié au virus de l'hépatite C en AMP
}

\author{
R. LEVY1, J.C. TARDY 2 , T. BOURLET ${ }^{3}$, H. CORDONIER 4 , J. LORNAGE4, J.F. GUERIN 4 .
}

1 Laboratoire de Biologie de la Reproduction. Hôpital Nord, 42055 SAINT ETIENNE

2 Laboratoire de Virologie, Domaine Rockefeller, 69373 LYON CEDEX 08

3 Laboratoire de Virologie, Hôpital Nord, 42055 SAINT ETIENNE

4 Laboratoire de Biologie de la Reproduction et du Développement., Hôpital Edouard Herriot, Place

d'Arsonval, 69437 LYON CEDEX 03

\section{RÉSUMÉ}

Nous sommes amenés à prendre en charge en assistance médicale à la procréation (AMP) un nombre croissant de couples dont l'un ou les deux partenaires présente (nt) une infection au virus de l'hépatite $\mathrm{C}$ (VHC). Le risque, jusqu'ici théorique, est la transmission du virus à l'enfant conçu en AMP, puis à sa mère, mais aussi celui de la contamination du personnel manipulant les gamètes infectés, ou des gamètes et embryons des couples noninfectés. Dans le but de mieux apprécier le risque de transmission du VHC en AMP via le sperme d'un patient infertile virémique, nous avons recherché le virus de l'hépatite $C(A R N$ VHC) par amplification génique (RT- PCR) dans le sperme et les différentes fractions obtenues après gradient de centrifugation. La recherche de l'ARN du VHC dans le sperme s'est révélée positive pour 2 patients (2/39) présentant une hépatite $C$ active. La détection de l'ARN VHC dans la fraction de spermatozoïdes mobiles obtenue après sélection en gradient de densité s'est révélée négative dans tous les cas.

Mots clés : ARN VHC, Assistance Médicale à la Procréation (AMP), Sperme, Virus de l'hépatite $C$ (VHC)

\section{INTRODUCTION}

Le risque de transmission d'un virus lors de la réalisation d'un acte d'Assistance Médicale à la Procréation (AMP) reste un sujet très débattu, en particulier dans le cas du virus de l'hépatite $C$. Les stratégies en matière de sécurité sanitaire en AMP varient considérablement d'un pays à l'autre [1]. En France, la loi de Bioéthique de Juillet 1994 a clairement défini le cadre des mesures de sécurité sanitaires en matière d'AMP avec tiers donneur, mais de simples recommandations existaient en matière d'AMP intra-couple. Le Guide de Bonnes Pratiques Cliniques et Biologiques en Assistance Médicale à la Procréation, publié au J.O. du 28-02-1999, précise ces recommandations et impose "la recherche de marqueurs biologiques d'infection, et lorsque cela est techniquement possible d'infectivité, par VIH 1, VIH 2, les virus des hépatites $B$ et $C$ et la syphilis » pour les deux membres du couples. Une séropositivité pour le VHC avec virémie positive de l'un ou des deux membres du couple interdit leur prise en charge en AMP, en dehors d'un protocole de recherche clinique pluridisciplinaire relevant de la loi Huriet, comprenant l'avis d'un CCPRB, et validé par la CNMBRDP. Depuis la parution des ce texte, aucun couple infertile dont l'un ou les deux membres présente une infection active pour le VHC n'a pu être pris en charge en AMP.

Le virus de l'hépatite $\mathrm{C}$ est un petit virus à ARN, d'environ 10000 nucléotides. 
Contrairement au VIH, le VHC ne possède pas l'équipement enzymatique (reverse transcriptase) permettant l'intégration de l'ADN viral dans les cellules infectées. Contrairement à l'hépatite $\mathrm{B}$, il n'existe à ce jour aucun vaccin contre l'hépatite $\mathrm{C}$, et aucune thérapeutique totalement efficace.

En France, la prévalence des anticorps antiVHC dans la population générale a été estimée à $1 \%$ en 1996 , ce qui correspond à 600000 personnes infectées par le VHC. Parmi ces sujets infectés, 400 à 500000 seraient virémiques pour le VHC [19], mais seuls 15 à 20000 cas ont été à ce jour diagnostiqués, et il n'est pas rare, lors du bilan avant AMP de découvrir une sérologie VHC.

Le mode de transmission du VHC est un mode essentiellement parentéral : la transfusion de produits sanguins contaminés ou une hémophilie (avant Mars 1990) sont incriminés dans environ $37 \%$ des cas ; $23 \%$ sont d'anciens toxicomanes ; $3,3 \%$ des cas impliquent clairement une contamination professionnelle ; $1,2 \%$, une transmission sexuelle (qui reste débattue); pour près de $15 \%$ des cas, une transmission nosocomiale a pu être identifiée avec certitude (hémodialyse, greffe). Les génotypes les plus répandus sont : $1 \mathrm{~b}$ (50\% des cas, en diminution, observé après transfusion), 1a et $3 \mathrm{a}$ (en augmentation, observé en cas de toxicomanie).

La transmission du VHC dans un contexte médical a été clairement démontrée : $46 \%$ des hépatites professionnelles à l'Assistance Publique des hôpitaux de Paris en 1991 ont été attribuées au VHC et des cas de séroconversion VHC ont été observés après blessures par aiguille en milieu hospitalier [8].

Le risque de transmission vertical du VHC varie d'une étude à l'autre : nul, en l'absence de virémie maternelle, faible (entre 3 et $8 \%$ ), mais encore à préciser, en cas de virémie élevée (>106 copies/ ml) [6, 18,19].

La présence du VHC dans différents liquides biologiques (salive, urines, ascite) a été affirmée par de nombreux auteurs [13, 24, 17] mais sa détection dans le sperme demeure controversée (Tableau 3). La présence d'inhibiteurs de la Taq Polymérase dans le sperme peut aboutir à des résultats non interprétables [20], voire à des faux négatifs.
Le risque viral en AMP, lorsque l'on considère un couple où l'homme est infecté et virémique pour le VHC, est la transmission du virus à l'enfant conçu en AMP, à la mère, mais aussi le risque nosocomial (contamination des gamètes et embryons de couples non infectés) et professionnel (personnes manipulant les gamètes potentiellement infectés, le jour de l'AMP ou en cas de cryoconservation) $[8,15]$.

Afin de préciser ce risque, nous avons recherché la présence de l'ARN VHC dans le sperme de 39 patients infertiles virémiques pour le VHC.

\section{MATÉRIEL ET MÉTHODES}

\section{Population étudiée}

39 patients infertiles âgés de 31 à 47 ans ont accepté de participer à cette étude. Tous étaient séronégatifs pour le VIH. Aucun n'était co-infecté par le virus de l'hépatite $B$. Tous les patients avaient des anticorps anti-VHC dans le sérum, et présentaient une infection active pour le VHC avec un ARN VHC détectable (AMPLICOR VHC, ROCHE) et quantifiable (MONITOR VHC, ROCHE SA, Neuilly /s/ Seine, France) dans leur sérum. Tous étaient asymptomatiques, mais présentaient des taux de transaminases élevés. Aucun patient n'était traité par des agents antiviraux au moment de l'étude: cependant, 3 patients avaient préféré retarder leur traitement (interféron $\alpha$ et ribavirine) pour bénéficier de ce protocole avec éventuelle congélation de sperme, avant mise sous ribavirine.

Linfection par le VHC a pu être attribuée à des produits sanguins ou une hémophilie dans 5 $(13 \%)$ et $3(7 \%)$ cas. 12 patients $(31 \%)$ avaient des antécédents de toxicomanie. Pour 3 patients $(7 \%)$, le seul élément d'orientation retrouvé à l'interrogatoire a été un mode de transmission nosocomial (1 greffe, 1 endoscopie, 1 hémodialyse). Aucun cas de transmission par voie sexuelle ou dans un cadre professionnel n'a été retrouvé. Pour 15 patients ( $42 \%$ ), le mode de contamination demeure inconnu.

Les paramètres spermatiques, déterminés selon les critères de l'OMS [25] (Tableau 1), montrent que 2 sujets présentaient une oligozoospermie et 10, une oligo-asthéno-tératozoo- 


\begin{tabular}{|c|c|c|c|c|c|}
\hline & $\begin{array}{l}\text { Volume de } \\
\text { l'éjaculat (ml) }\end{array}$ & $\begin{array}{l}\text { Concentration } \\
\text { (x 106/ml) }\end{array}$ & $\begin{array}{l}\text { Mobilité } \\
(\text { type a }+\mathbf{b})(\%)\end{array}$ & $\begin{array}{l}\text { Morphologie } \\
\text { (\% anormaux) }\end{array}$ & $\begin{array}{l}\text { Leucocytes } \\
106 / \mathrm{ml}\end{array}$ \\
\hline $\begin{array}{l}\text { Moyenne } \\
(n=39)\end{array}$ & 3.0 & 52.4 & 34.3 & 50.6 & 0.6 \\
\hline Intervalle & $0.3-8.5$ & $1.80-346$ & $5-55$ & $12-86$ & $0-6.4$ \\
\hline
\end{tabular}

Tableau 2 : Détection de l'ARN VHC dans le sperme des patients infertiles (HCV - AMPLICOR).

\begin{tabular}{|c|c|c|c|c|}
\hline Echantillons testés & $\begin{array}{l}\text { DO } \mathbf{A}_{450} \\
\text { Sans CI }\end{array}$ & $\begin{array}{l}\text { DO A450 } \\
\text { Avec CI }\end{array}$ & $\begin{array}{l}\text { DO A450 } \\
\text { Sans CI } \\
\text { Après dilution } \\
\text { au } 1 / 4\end{array}$ & $\begin{array}{l}\text { DO A450 } \\
\text { Avec CI } \\
\text { Après dilution } \\
\text { au } 1 / 4\end{array}$ \\
\hline Contrôle négatif & 0.06 & 0.91 & & \\
\hline Contrôle positif & 0.84 & 0.92 & & \\
\hline Patient $N^{\circ} 1$ : Sperme & 0.06 & $0.05 *$ & 0.07 & $>\mathbf{3}$ \\
\hline Culot cellulaire & 0.06 & 0.91 & & \\
\hline Plasma séminal & 0.05 & $0.05 *$ & 0.08 & $>3$ \\
\hline $50 \%$ après Percoll & 0.06 & 0.84 & & \\
\hline $70 \%$ après Percoll & 0.06 & $0.8390 \%$ & & \\
\hline après Percoll & 0.06 & 0.85 & & \\
\hline Patient $N^{\circ} 35$ : Sperme & 2.71 & $>\mathbf{3}$ & & \\
\hline Culot cellulaire & 2.86 & 2.96 & & \\
\hline Plasma séminal & 1.99 & 2.79 & & \\
\hline $50 \%$ après Percoll & 0.09 & 2.76 & & \\
\hline $70 \%$ après Percoll & $0.08>3$ & $90 \%$ & & \\
\hline après Percoll & 0.08 & 2.91 & & \\
\hline Patient $N^{\circ} 6$ : Semen & 0.07 & $0.10 *$ & 0.10 & 2.77 \\
\hline Culot cellulaire & 0.72 & $>3$ & & \\
\hline Plasma séminal & 0.08 & $0.06 *$ & 2.74 & 2.92 \\
\hline $50 \%$ après Percoll & 0.08 & 2.85 & & \\
\hline $70 \%$ après Percoll & 0.08 & 2.54 & & \\
\hline $90 \%$ après Percoll & 0.08 & 2.74 & & \\
\hline
\end{tabular}

Présence d'inhibiteurs de la Taq polymérase

Kit de détection AMPLICOR VHC : DO A450

$<0.25=$ résultat négatif $; 0.25-0.6=$ non interprétable $;>0.6=$ résultat positif

Kit avec Contrôle Interne (CI) AMPLICOR:

$\begin{array}{lll}\mathrm{DO} \mathrm{A}_{450} & \mathrm{DO} \mathrm{A}_{450}+\mathrm{VHC} \mathrm{CI} & \\ <0.25 & >0.6 & \text { Résultat négatif } \\ & & \text { Pas d'inhibiteurs } \\ <0.25 & <0.6 & \begin{array}{l}\text { Non interprétable } \\ \text { Présence d'inhibiteurs }\end{array}\end{array}$


spermie. Lindication de FIV reposait sur un facteur masculin dans $30 \%$ des cas, féminin dans $40 \%$ des cas et mixte dans $10 \%$ des cas. Six couples avaient déjà un enfant, né hors AMP et non infecté par le VHC.

\section{a) Détection du VHC.}

Après liquéfaction de l'éjaculat recueilli dans un flacon stérile, un millilitre de sperme $\left(\mathrm{N}^{\circ} 1\right)$ a été prélevé. Un autre millilitre de sperme a été centrifugé $(800 \mathrm{~g} \times 10 \mathrm{~min})$ : le plasma séminal $\left(\mathrm{N}^{\circ} 3\right)$ a été séparé du culot cellulaire $\left(\mathrm{N}^{\circ} 2\right)$. Un millilitre a été déposé sur un gradient de densité (50,70 et 90\%) (Percoll, Pharmacia, Uppsala) pour sélectionner les spermatozoïdes mobiles. Chaque fraction obtenue a ensuite été lavée et le culot remis en suspension dans $500 \mu \mathrm{l}$ de BM1 (BM1®, Ellios Bio Media) (fractions $\mathrm{N}^{\circ} 4,5,6$ et 7 ). Les spermatozoïdes présents dans les différentes fractions ont été comptés avant congélation à $-80^{\circ} \mathrm{C}$. Ces différentes étapes ont été effectuées en moins de deux heures.

Extraction de l'ARN VHC : La technique d'extraction utilisée a été la technique classiquement recommandée dans le kit VHC AMPLICOR HCV (ROCHE SA, Neuilly /s/Seine, France).

$R T-P C R$ : Les échantillons de sperme ont tous été testés avec le kit commercialisé pour l'amplification et la détection de l'ARN VHC (VHC AMPLICOR Amplification Kit et VHC Detection Kit ; ROCHE SA, Neuilly/s/Seine, France). Tous les échantillons ont été testés avec un contrôle négatif et un contrôle positif pour éliminer toute contamination. La sensibilité de la technique est évaluée à 200 copies du génome $\mathrm{VHC} / \mathrm{ml}$.

Détection des inhibiteurs de la Taq polymérase : Nous avons utilisé le kit AMPLICOR avec contrôle interne (AMPLICOR Internal Control (IC) Detection Kit (ROCHE SA, Neuilly /s/Seine, France) ). Ce kit a été associé à une méthode de détection en plaques (AMPLICOR Microwell Plate (MWP)) avec lecture des résultats en densité optique (DO). Un contrôle interne a été introduit dans chaque tube et co-amplifié avec l'ARN testé. Un résultat négatif $(<0.25)$ après addition du contrôle interne indique la présence d'inhibiteurs de la
Taq polymérase dans l'échantillon et empêche la validation $\mathrm{du}$ résultat. La dilution de l'échantillon au $1 / 4$ a permis de diluer les inhibiteurs, d'obtenir des données interprétables pour tous les patients et d'éviter les faux négatifs.

\section{RÉSULTATS}

Pour deux des trente neuf patients infertiles et virémiques pour le VHC, l'ARN VHC a pu être détecté dans le sperme (Tableau 2). Dans un cas $\left(n^{\circ} 35\right)$, l'ARN VHC a été clairement détecté dans l'éjaculat, le plasma séminal et le culot cellulaire, en l'absence d'inhibiteurs. Le patient avait été contaminé en 1982 (toxicomanie). L'analyse du sperme a montré une oligoasthénotératozoospermie sévère avec 240 000 leucocytes. La quantification de l'ARN VHC a permis de retrouver $8,7.10^{4}$ copies/ $\mathrm{ml}$ dans le sérum $(4,93 \mathrm{log}$ copies/ $\mathrm{ml})$ et 7,5. 102 copies/ $\mathrm{ml}$ dans le plasma séminal (2,88 log copies/ $\mathrm{ml}$ ). Un génotype identique, le génotype $3 \mathrm{a}$, a été retrouvé dans le sérum du patient et le plasma séminal.

Pour le second patient $\left(\mathrm{n}^{\circ} 6\right)$, la présence d'inhibiteurs dans les fractions $\mathrm{N}^{\circ} 1$ (éjaculat) et $\mathrm{N}^{\circ} 3$ (plasma séminal) ne permettait pas l'obtention d'un résultat interprétable : après dilution au $1 / 4$, l'analyse des fractions $\mathrm{N}^{\circ} 1$ et $\mathrm{N}^{\circ} 3$ a permis la détection de l'ARN VHC dans le plasma séminal $\left(\mathrm{N}^{\circ} 3\right)$. La contamination par le VHC datait de 1970 (toxicomanie). Les caractéristiques du sperme étaient normales et on notait l'absence de leucocytes. La quantification de l'ARN VHC a permis de retrouver 1,7.106 copies/ $\mathrm{ml}$ dans le sérum $(6,23 \mathrm{log}$ copies/ $\mathrm{ml}$ ) et 2,4 $10^{3}$ copies/ $\mathrm{ml}$ dans le plasma séminal (3,38 log copies/ $\mathrm{ml})$. Le génotype 1a a été déterminé dans le sérum du patient et dans le plasma séminal.

Des inhibiteurs de la Taq polymérase ont pu être détectés par notre méthode dans près de $80 \%$ des cas dans le sperme et le plasma séminal $\left(\mathrm{N}^{\circ} 1\right.$ et $\left.\mathrm{N}^{\circ} 3\right)$, mais jamais dans le culot cellulaire $\left(\mathrm{N}^{\circ} 2\right)$, ni dans les fractions après gradient de Percoll ( $\left.\mathrm{N}^{\circ} 4,5,6,7\right)$. Toutes les fractions $90 \%$ obtenues après Percoll et contenant les spermatozoïdes mobiles $\left(\mathrm{N}^{\circ} 7\right)$ se sont révélées négatives.

L'ARN VHC a pu être quantifié dans le sérum 
des patients infertiles dans $72 \%$ des cas et montre un intervalle de valeurs comprises entre 5331 et 5.106 copies/ $\mathrm{ml}$. Dans les deux cas où la recherche de l'ARN VHC dans le sperme s'est révélée positive, une forte charge virale plasma tique était présente. Dans les deux cas, une différence de deux logarithmes a pu être observé entre la charge virale VHC dans le sérum et la charge virale dans le plasma séminal.

Deux patients dont la recherche de l'ARN VHC dans le sperme était négative ont bénéficié d'un contrôle à 1 mois d'intervalle, qui s'est révélé à nouveau négatif. Les deux patients pour lesquels l'ARN VHC avait été détecté dans le sperme ont eux aussi bénéficié d'un contrôle à 6 mois d'intervalle : dans un cas, l'ARN VHC a été détecté dans la même fraction et en quantité identique après dilution au $1 / 4$ (patient $n^{\circ} 6$ ); dans le second cas $\left(n^{\circ} 35\right)$, la recherche s'est révélée négative.

\section{DISCUSSION}

En France, les mesures de sécurité sanitaire définissent de façon stricte le bilan avant AMP intra-couple : sérologies VIH 1, VIH 2 , hépatites B et C, syphilis. Depuis le 28-02-1999, la découverte d'une sérologie positive pour le VHC aboutit, en cas d'infection active, à l'exclusion du patient de tout programme d'AMP.

De nombreuses études rassurantes ont affirmé l'absence totale de VHC dans le sperme [7, 5, $23,2,20]$; d'autres affirment au contraire que le VHC peut être détecté dans le sperme avec une prévalence très faible [4] ou forte $[9,10$, $13,14,2]$ (Tableau 3). Ces résultats contradictoires peuvent être expliqués par des différences dans (1) la population étudiée (co-infection par le VIH), (2) l'obtention et la conservation des échantillons (congélation rapide à $80^{\circ} \mathrm{C}$ ), (3) la sensibilité des techniques utilisées et (4) la détection des inhibiteurs de la Taq polymérase (RNases ou lipopéroxidases qui interférent avec l'amplification des acides nucléiques et pourraient expliquer l'absence de détection de l'ARN VHC dans le sperme). Notre étude retrouve de tels inhibiteurs dans près de $80 \%$ des spermes et plasma séminaux testés, mais dans aucune des fractions obtenues après centrifugation sur gradient de den- sité. La simple dilution au $1 / 4$ des spermes et plasma séminaux avec inhibiteurs a permis d'obtenir des résultats interprétables dans tous les cas. Cependant, le fait de diluer nos échantillons est critiquable : afin de maintenir la sensibilité de notre technique, nous testons d'autres techniques d'extraction des ARN utilisant des particules de silice permettant d'éliminer les inhibiteurs enzymatiques [21].

Notre étude a permis de détecter l'ARN VHC dans le sperme dans 2 cas. Pour l'un des patients, l'absence d'inhibiteurs dans les échantillons analysés a permis la détection de l'ARN VHC sans étape de dilution préalable ; l'ARN VHC a été détecté dans le sperme, le plasma séminal et le culot cellulaire obtenu après centrifugation et contenant les spermatozoïdes, les leucocytes et les cellules rondes. Dans le second cas, le plasma séminal était négatif avant dilution, mais avec présence d'inhibiteurs ; l'ARN VHC a été détecté dans le plasma séminal après dilution au $1 / 4$. Dans les deux cas, il s'agissait de patients avec des antécédents de toxicomanie et présentant une infection ancienne par le VHC (>20ans). Dans les deux cas, la charge virale VHC dans le sérum était élevée (8,7 $10^{4}$ et $1,710^{6}$ copies/ $\mathrm{ml}$ ) et une différence de deux logarithmes a pu être observée entre la charge virale VHC dans le sérum et dans le plasma séminal $\left(7,510^{2}\right.$ et $2,410^{3}$ copies / ml).

Tous les tests effectués sur les fractions obtenues après centrifugation en gradient de densité se sont révélés négatifs avec absence d'inhibiteurs. Nos résultats corroborent l'étude de Mc Kee et al. [16], qui note l'absence d'ARN VHC après centrifugation en gradient de densité d'un sperme de donneur positif en ARN VHC. Les différentes étapes de la préparation de sperme (sélection des spermatozoïdes mobiles par centrifugation sur gradient de densité avec élimination du plasma séminal, des leucocytes, cellules rondes et spermatozoïdes immobiles et peu mobiles ; puis lavage de la fraction $90 \%$ ) peuvent expliquer l'élimination (ou la forte diminution) de l'ARN VHC après centrifugation en gradient de densité. Ainsi, même si l'étape de sélection en gradient de densité peut être techniquement supprimée avant ICSI, et ce sans aucun effet péjoratif sur 


$\begin{array}{ll}\begin{array}{l}\text { Technique } \\ \text { utilisée }\end{array} & \begin{array}{l}\text { Résultats } \\ \text { dans le sperme }\end{array}\end{array}$

Semprini et al. 1998

RT - PCR

Caldwell et al. 1996

Tang et al. 1996

McKee et al. 1996

Fiore et al. 1995

Liu et al. 1994

Liou et al. 1992

Fried et al.1992

Hsu et al. 1991

Terada et al. 1991

$\begin{array}{ll}\text { RT - PCR } & \text { Négative } \\ \text { RT - PCR } & \text { Positive (57\%) } \\ \text { RT - PCR } & \text { Positive }\end{array}$

RT - PCR

RT - PCR

RT - PCR

nested

RT - PCR

nested PCR

RT - PCR

nested PCR

RT - PCR

nested PCR

Positive

Positive

Négative

Négative

Négative
Négative

\section{Population étudiée}

90 sujets toxicomanes dont 56 patients virémiques pour le VHC, 27 co - infectés VIH et $\mathrm{VHC}$

12 patients virémqiues

7 patients virémiques

Donneur de sperme ARN VHC négatif après Percoll

PCR Positive (24\%)

3 patients virémiques

34 patients virémiques

14 patients virémiques

19 patients virémiques les taux de fécondation et de clivage embryonnaire [3], nous considérons que, chaque fois que les caractéristiques du sperme le permettent, une centrifugation en gradient de densité est préférable. Cette technique simple, rapide et peu onéreuse élimine (ou diminue) divers agents infectieux tels le Cytomegalovirus [11], Chlamydia trachomatis [12] ou le VHC.

Notre étude montre que la détection de l'ARN VHC dans le sperme est possible. Différentes techniques d'extraction des ARN doivent maintenant être testées dans le sperme afin de permettre la validation et la standardisation d'une technique de détection virale. Pour les couples infertiles dont l'homme présente une infection active pour le VHC, le bilan biologique doit être complété par la recherche de l'ARN VHC dans le sérum. En l'absence de virémie, l'AMP pourra être réalisée sans délai. En cas de virémie positive, après quantification de la charge virale dans le sérum, la détection de l'ARN VHC dans le sperme, avant et après sélection des spermatozoïdes (et si positive, sa quantification) sera effectuée. Pour plus de sécurité, l'AMP pourra alors être réali- sée en utilisant des paillettes de sperme congelé préalablement testé et négatif.

Dans tous les cas, une information précise et détaillée des techniques utilisées, de leurs risques et limites et des résultats attendus doit être fournie au couple et leur consentement écrit doit être recueilli. Enfin, la manipulation de spermes potentiellement infectés et la réalisation de l'AMP au sein du laboratoire requiert des mesures de précaution sanitaires particulièrement strictes : utilisation de matériel jetable à usage unique, hottes à flux vertical, gants, masque, blouse ainsi que des protocoles de désinfection des appareils et des étuves appropriés.

\section{REFERENCES}

1. ABUSHEIKHA, N., AKAGBOSU, F., MARCUS, S., COUSINS, C., BRINSDEN, P. Viral screening and Assisted Conception Treatment - The Bourn Hall Experience. J. Assist. Reprod. Genet. 1999, 16, 337339.

2. CALDWELl, S. H., SUE, M., BOWDEN, J. H., DICKSON, R.C., DRISCOLL, C.J., YEATON, P., STEVENSON, W.C., ISHITANI, M.B., MC 
CULLOUGH, C.S., PRUETT, T.L., LOVELL, M.A. : Hepatitis $\mathrm{C}$ virus in body fluids after liver transplantation. Liver Transpl. Surg.1996, 2 (2), 124-129.

3. DE VOS, A., NAGY, Z.P., VAN DE VELDE, H., JORIS, H., BOCKEN, G., VAN STEIRTEGHEM, A. Percoll gradient centrifugation can be omitted in sperm preparation for intracytoplasmic sperm injection. Hum. Reprod. 1997, 12, 1980-1984.

4. FIORE, R. J., POTENZA, D., MONNO, L., APICE, A., DISTEPHANO, M., GIANNELLI, A., LAGRASTA, L., ROMANELLI, C., DIBARI, C., PASTORE, G. : Detection of HCV RNA in serum and seminal fluid from HIV - 1 co - infected intravenous drug addicts. J. Med. Virol.1995, 46, 364-367.

5. FRIED, M. W., SHINDO, M., FONG, T., FOX, P. C., HOOFNAGLE, J. H., DI BISCEGLIE, A. : Absence of hepatitis C viral RNA from saliva and semen of patients with chronic hepatitis C. Gastroenterology, 1992, 102, 1306-1308.

6. GILLET, P., HALLAM, N., MOK, J. Vertical transmission of hepatitis $\mathrm{C}$ virus infection. Scand. J. Infect. Dis., 1996 , 28, 549-552.

7. HSU, H. H., WRIGHT, T., L., LUBA, D., MARTIN, M., FEINSTONE, S. M., GARCIA, G., GREENBERG, H. B. : Failure to detect hepatitis $\mathrm{C}$ virus genome in human secretions with the polymerase chain reaction. Hepatology, 1991, 14, 763-767.

8. KIYOSAWA, K., SODEYAMA, T., TANAKA, E. : Hepatitis $\mathrm{C}$ in hospital employees with needlestick injuries. Ann. Intern. Med. 1991, 115, 367-369.

9. KOTWAL, G. J., RUSTGI, V. K., BAROUDY, B. M. :Detection of hepatitis $C$ virus specific antigens in semen from non A, non B hepatitis patients. Dig. Dis. Sci.,1992, 35, 641-644.

10. KOTWAL, G. J. : Routine laboratory diagnosis of hepatitis C virus infection. J. Hepatol. 1993, 17, Suppl. 3, S83-S89.

11. LEVY, R., NAJIOULLAH, F., KEPPI, B., THOUVENOT, D., BOSSHARD, S., LORNAGE, J., LINA, B., GUERIN, J. F., AYMARD, M. : Detection of cytomegalovirus in semen from a population of men seeking infertility evaluation. Fertil. Steril.1997, 68, 820-826.

12. LEVY, R.,LAYANI-MILON, M. P., GISCARD D'ESTAING, S., NAJIOULLAH, F., LORNAGE, J., AYMARD, M. AND LINA, B. : Screening for Chlamydia trachomatis and Ureaplasma urealyticum infection in semen from asymptomatic male partners of infertile couples prior to in vitro fertilization. Int. J. Androl., 1999 , 22, 113-118.

13. LIOU, T., CHANG, T. T., YOUNG, K. C., LIN, X. Z., LIN, C. Y., WU, H. L. :Detection of HCV RNA in saliva, urine, seminal fluid and ascites. J. Med. Virol.1992, 37, 197-202.

14. LIU, F. H., TIAN, G. S., FU, X. X. : Detection of plus and minus strand hepatitis $\mathrm{C}$ virus RNA in periphe- ral blood mononuclear cells and spermatid, Abstract, Chinese Medical Journal,1994, 74 (5), 284-6.

15. MASSEY, E.J., DASANI, H., JONES, P., SALEEM, A.K.N. : Storage facility exists for sperm from patients positive for antibody to hepatitis $\mathrm{C}$ virus. B.M.J.1996, 313 : 1078.

16. MC KEE, T. A., AVERY, S., MAJID, A., BRINSDEN, P. R. : Risks for transmission of hepatitis $C$ virus during artificial insemination. Fertil. Steril.1996, 66 (1) : 161-163.

17. NUMATA, N., OHORI, H., HAYAKAWA, Y., SAITOH, Y., TSUNODA, A., KANNO, A. : Demonstration of hepatitis $C$ virus genome in saliva and urine of patients with type $C$ hepatitis : usefulness of the single round polymerase chain reaction method for detection of the HCV genome. J. Med. Virol. 1993, 41: 120-128.

18. OHTO, H., TERAZAWA, S., SASAKI, N., SASAKI, N., HINO, K., ISHIWATA, C., KAKO, M., UJIIE, N., ENDO, C., MATSUI, A., OKAMOTO, H., MISHIRO, S. : Transmission of hepatitis $\mathrm{C}$ virus from mothers to infants. New Engl J Med1994, 330 : 744-750.

19. Roudot - Thoraval, F., Pawlotsky, J. M., Thiers, V., DESFORGES, L., GIROLLET, P. P., GUILLOT, F., HURAUX, C., AUMONT, P., BRECHOT, C., DHUMEAUX, D. : Lack of transmission of hepatitis $\mathrm{C}$ virus in human immunodeficiency virus seronegative women : a prospective study with hepatitis $\mathrm{C}$ virus RNA testing. Hepatology,1993, 17, 772-777.

20. SEMPRINI, A. E., PERSICO, T., THIERS, V., ONETA, M., TUVERI, R., SERAFINI, P., BOSCHINI, A., GIUNTELLI, S., PARDI, G., BRECHOT, C. : Absence of hepatitis $\mathrm{C}$ virus and detection of hepatitis G virus / GB virus C RNA sequences in the semen of infected men. J. Infect. Dis. 1998, 177 (4), 848-854.

21. TACHET, A., DUlioust, E., SAlMON, D., DE ALMEIDA, M., RIVALLAND, S., FINKIELSZTEJN, L., HEARD, I., JOUANNET, P., SICARD, ROUZIOUX, C. : Detection and quantification of HIV-1 in semen : identification of a subpopulation of men at high potential risk of viral sexual transmission. AIDS, 1999, 7, 823-831.

22. TANG, Z., YANG, D., HAO, L., TANG, Z., HUANG, Y., WANG, S. : Detection and significance of HCV RNA in saliva, seminal fluid and vaginal discharge in patients with hepatitis C. J. Tongji Med. Univ.1996, 16 (1), 11-13.

23. TERADA, S., KAWANISHI, K., KATAYAMA, K. : Minimal hepatitis $\mathrm{C}$ infectivity in semen. Annals Intern. Med., 1992, 117, 171-172.

24. YOUNG, K., CHANG, T., LIOU, T., WU, H. : Detection of hepatitis $\mathrm{C}$ virus RNA in peripheral blood mononuclear cells and in saliva. J. Med. Virol., 1993, 41, 55-60.

25. World Health Organization (1992) WHO Laboratory Manual for the Examination of Human Semen and Sperm - Cervical Mucus Interaction. Cambridge University Press. 


\section{ABSTRACT \\ Transmission risk of $\mathrm{c}$ hepatitis virus in assisted reproductive techniques \\ R. LEVY, J.C. TARDY, T. BOURLET, H. COR- DONIER, J. LORNAGE, J.F. GUERIN.}

Medical assistance to procreation in a couple where one or both parents has hepatitis $C$ viral infection $(\mathrm{HCV})$ raises the issue of the transmission of the infection to the baby and/or of possible contamination of both the technicians and the gametes or embryos from virus-free parents in the laboratory. It becomes essential to assess transmission risk in Assisted Reproductive Techniques (ART) in order to clearly define the management of couples according to their viral status. To define the HCV transmissibility risk in ART related to the presence of virus in semen from infected infertile men, RNA HCV-detection was performed in sera, and semen and sperm fractions obtained after Percoll gradient centrifugation. RNA HCV was detected in 5\% (2 / 39) of the semen tested : in the raw semen, in the seminal fluid and in the cell pellet but never after Percoll selection. According to these results, we suggest a strategy for $\mathrm{HCV}$ infected infertile men who need ART.

Key words : Assisted Reproductive techniques, Hepatitis C Virus, Semen, Viral Screening 